\title{
Bacteriome and Mycobiome in Nicotiana tabacum Fields Affected by Black Shank Disease
}

\author{
Xiaoyulong Chen, ${ }^{1,2}$ Baoyu He, ${ }^{2}$ Hong Yang, ${ }^{1,2}$ and Tomislav Cernava ${ }^{1,2,3, \dagger}$ \\ ${ }^{1}$ College of Tobacco Science, Guizhou University, 550025, Guiyang, China \\ ${ }^{2}$ Guizhou Provincial Key Laboratory for Agricultural Pest Management of the Mountainous Region, \\ 550025, Guiyang, China \\ ${ }^{3}$ Institute of Environmental Biotechnology, Graz University of Technology, 8010 Graz, Austria
}

\begin{abstract}
Phytophthora nicotianae is a widespread cause of black shank disease of tobacco plants and causes substantial harvest losses in all major cultivation areas. The oomycete primarily affects plant roots and the stem, where it leads to a progressing decay of the diseased tissues. In this resource announcement, we provide two complementary datasets comprising $16 \mathrm{~S}$ gene fragment amplicons (bacteriome) and ITS1 region amplicons (mycobiome) that were sequenced on an lllumina-based platform. Soil samples were obtained from disease-affected fields in Guizhou province (China) and include control samples from adhering fields without previous disease incidence. Both datasets were acquired at a high sequencing depth and accompanied by detailed metadata, which facilitate their implementation in comparative studies. The resource announcement provides a basis for disease-specific biomarker detection and correlation studies that include the microbiome.
\end{abstract}

\section{Background and Context for the Resource}

The cultivation of Nicotiana tabacum L. has a long tradition in human history. Its seeds were recovered from historic settlements (1500 to $1000 \mathrm{BC}$ ), providing evidence for tobacco cultivation during the early Chiripa phase in South America (Groark 2010). Nowadays, China, Brazil, India, and the United Sates, ordered according to their yearly production capacities, harbor the largest cultivation areas and are the main producers of $N$. tabacum leaves (FAO 2019). Within China, Guizhou province harbors the second largest cultivation area for tobacco plants and is only surpassed by Yunnan province (Wang et al. 2013).

Similar to other plants from the Solanaceae family, various viruses, nematodes, and soilborne pathogens can account for the highest yield losses (Ma et al. 2018). Phytophthora nicotianae Breda de Haan is one of these devastating pathogens and causes necrotic black shank disease in N. tabacum. The disease was first reported in 1896 in Indonesia (Shew and Lucas 1991). Since 1915, the disease was widely reported in the United States and since 1950 in China (Erwin and Ribeiro 1996). P. nicotianae has a broad range of hosts and can infect plants from more than 90 families including 255 genera (Panabieres et al. 2016). Owing to its widespread occurrence and devastating effects, exploration of effective and sustainable management strategies is urgently needed.

Recently, the tobacco soil microbiome was explored under different incidences of bacteria wilt caused by Ralstonia solanacearum (Liu et al. 2016; Wu et al. 2014; Yang et al.

\footnotetext{
${ }^{\dagger}$ Corresponding author: T. Cernava; tomislav.cernava@tugraz.at

*The $e$-Xtra logo stands for "electronic extra" and indicates that four supplementary figures and two supplementary tables are published online.
}

The author(s) declare no conflict of interest.

Accepted for publication 10 September 2019.
Funding

This study is supported by the National Natural Science Foundation of China (grant nos. 31960555, 31701836), Science and Technology Foundation of Guizhou Province (grant nos. 20175788, 2018-1050, 2019-1410), Guizhou Tobacco Company Research Program (grant no. 201626), and Guizhou Qianxinan Tobacco Company Research Program (grant no. 201804).

\section{Keywords}

Phytophthora nicotianae, oomycetes, Solanaceae, microbiome, biomarkers 
2017). The authors concluded that the soil microflora plays an important role in the bacteria wilt incidence as well as its management (Jiang et al. 2017; Shen et al. 2018). In order to holistically understand how the soil microbiome influences and interacts with the plant, implementation of different experimental approaches, including meta-analyses of large microbial datasets related to the plant health, defense, and disease, will be required (Ginnan et al. 2018). In this context, no studies based on next-generation sequencing (NGS) approaches are available yet to investigate if soil microbial communities contribute to the development, incidence, and management of $P$. nicotianae during tobacco cultivation.

The aim of the study was to utilize complementary NGS methods to provide bacteriome and mycobiome datasets from field soils with black shank disease incidence. Moreover, reference samples from adhering fields with no disease incidence were obtained for comparisons. Samplings were conducted at three distinct geographical regions with large tobacco cultivation areas in Guizhou province, China. Our data could provide the basis to develop new black shank disease management strategies and early detection approaches in the future.

\section{Methods}

Sample description and experiment design. The samples were obtained from three distinct tobacco cultivation areas in Guizhou province (China) on November 15, 2018. The sampling sites were selected according to black shank disease occurrence and were located in An'long (25.28 N, 105.29 E), Xingren (25.35 N, 105.10 E), and Xingyi (25.20 N, 105.05 E). From each field, six soil samples (700 to $800 \mathrm{~g}$ per sample) were obtained postharvest from the topsoil layer ( 1 to $10 \mathrm{~cm}$ depth) and in radial distances of 5 to $10 \mathrm{~cm}$ from $N$. tabacum stems. The samples were stored in isolated boxes at $4^{\circ} \mathrm{C}$ until arrival in the laboratory (Guizhou Provincial Key Laboratory for Agricultural Pest Management of the Mountainous Region, Guizhou University, Guiyang, China). From each sampling site, $550 \mathrm{~g}$ of soil was analyzed by the laboratory of Xi'an United Nations Quality Detection Technology Co. (Xi'an, China) in terms of physicochemical properties.

Total community DNA extraction. From each of the samples, $100 \mathrm{mg}$ of soil was weighed and directly transferred to tubes containing extraction buffer (MP Biomedicals, Santa Ana, CA). The total community DNA was extracted with the FastDNA SPIN Kit for Soil (MP Biomedicals) following the manufacturer's protocol. Subsequently, samples were analyzed with NanoDrop (Thermo Fisher Scientific, Waltham, MA) to confirm the extraction efficiency.

Barcoded amplicon sequencing of 16S rRNA gene fragments. The samples were amplified with the primers 515f (5'-GTGYCAGCMGCCGCGGTAA) and 806r (5'-GGACTACHVGG GTWTCTAAT) according to the Earth Microbiome Project protocol (www.earthmicrobiome.org) with sample-specific barcodes and Illumina sequencing adaptors. The sequencing was conducted on an Illumina PE250 platform ( $2 \times 250$ bp paired-end reads) by Novogene (Beijing, China).

Barcoded amplicon sequencing of ITS1 region fragments. The same DNA extract was used for sequencing of the ITS1 region with primers ITS1f (5'-CTTGGTCATTTAGAGG AAGTAA) and ITS2 (5'-GCTGCGTTCTTCATCGATGC) according to the Earth Microbiome Project protocol (www.earthmicrobiome.org) with sample-specific barcodes and Illumina sequencing adaptors. The sequencing was conducted on an Illumina PE250 platform ( $2 \times$ 250 bp paired-end reads) by Novogene.

Bioinformatic analyses. The datasets were processed with a standardized workflow for further dereplication and quality filtering. In order to demultiplex the 16S rRNA gene fragment library, paired-end reads were assigned to samples based on their unique barcode and truncated by removing their barcode and primer sequence from the raw reads. Corresponding paired-end reads were merged using FLASH version 1.2.7 (Magoč and Salzberg 2011) before further bioinformatic processing. Subsequently, quality filtering of the raw tags was performed under strict filtering conditions to obtain high-quality tags (Bokulich et al. 2013). The resulting output was used to identify and remove all chimeric sequences using the UCHIME algorithm (Edgar et al. 2011; Haas et al. 2011). The taxonomic analysis was performed after the sequences were clustered at $97 \%$ similarity with UPARSE software (Edgar 2013). The assignments were based on a naïve-Bayes RDP classifier (Wang et al. 2007) clustered at $97 \%$ similarities with the SILVA 128 database (https://www.arb-silva.de/) for 16S rRNA gene fragments and the UNITE 8.0 database (https://unite.ut.ee/) for the fungal ITS1 region. Statistical significance of alpha diversity (Shannon index) analyses was assessed either with ANOVA and Tukey's post hoc test $(P<0.05)$ or with the Mann-Whitney $U$ test $(P<0.05)$ when two sample groups were compared. 
Detection of $\boldsymbol{P}$. nicotianae in total community DNA extracts. The same samples that were used to generate the amplicon libraries were also used to evaluate if $P$. nicotianae was present in the DNA extracts. The assessment was conducted with a qPCR-based method developed by Huang and colleagues (2010). Briefly, the primer pair P.nic1 (5'-GAAC AATGCAACTTATTGGACGTTT-3') and P.nic2 (5'-AACCGAAGCTGCCACCCTAC-3') was used in combination with PowerUp SYBR Green Master Mix (Applied Biosystems, Vilnius, Lithuania). All samples were adjusted to a concentration of $50 \mathrm{ng} / \mu \mathrm{l}$ according to NanoDrop 2000 (Thermo Scientific, Wilmington, DE) quantifications and subsequently processed with a CFX96 Real-Time System (Bio-Rad Laboratories, Hercules, CA). Each sample was analyzed with eight technical replicates.

\section{Results}

The resource announcement provides two NGS libraries with 16S rRNA gene fragment and ITS region amplicons from tobacco cultivation field soils with and without disease incidence. So far, black shank disease of $N$. tabacum was not addressed by microbiome studies targeting bacterial and/or fungal markers in field soils. We have subjected the datasets to a general bioinformatic analysis for a first assessment of their microbial diversity and taxonomic composition. Deepening bioinformatic analyses and comparisons with other NGS datasets will be required to assess disease-specific occurrence of distinct bacterial and fungal taxa in the field soils.

Bacterial diversity and community structure. The 16S rRNA gene fragment library was rarefied at 123,346 reads per sample. Alpha diversity was assessed for samples grouped according to the three sampling sites as well as for samples grouped according to the disease status. When the different sampling locations were compared, the alpha diversity was highly similar (Supplementary Figure S1). The highest Shannon index $(\mathrm{H})$ was observed for fields in Xingyi $(H=10.6)$ followed by fields in An'long $(H=10.5)$ and fields in Xingren $(H=10.2)$. When the samples were grouped according to occurrence of black shank disease, both groups had a Shannon index of 10.4. Moreover, no significant differences in alpha diversity were detected when Tukey's test $(P<0.05)$ and the Mann-Whitney $U$ test $(P<0.05)$ were applied. The observed species (97\% threshold for operational taxonomic unit [OTU] picking) ranged between 6,065 (sample FCB2 from An'long) and 8,426 (sample FAB5 from Xingyi). The first assessment of the community structure was conducted on family level owing to the high diversity of the bacteriome (Supplementary Figure S2). Gemmatimonadaceae (average abundance across all datasets $4.1 \%$ ) was the predominant family followed by Acidobacteriaceae $(2.4 \%)$, an unidentified family of Subgroup $6(2.3 \%)$, Sphingomonadaceae $(1.9 \%)$, and Xanthomonadaceae $(1.8 \%)$.

Fungal diversity and community structure. In a complementary approach, the fungal diversity was assessed in the same field soil samples that were used for bacteriome analyses. For the fungal amplicon library, the rarefaction was conducted at 51,622 reads per sample. The alpha diversity was assessed for samples grouped according to the three sampling sites as well as for samples grouped according to the disease status. The diversity within the mycobiome was generally lower than in the bacteriome (Supplementary Figure S3). Analogous to bacterial diversity, the highest Shannon index was observed for fields in Xingyi $(\mathrm{H}=$ 5.8) followed by An'long $(H=5.2)$ and Xingren $(H=5.0)$. The fungal diversity was lower in black shank disease-affected samples $(H=5.2)$ compared with samples obtained from fields without disease occurrence $(\mathrm{H}=5.5)$. No significant differences in alpha diversity were detected when Tukey's test $(P<0.05)$ and the Mann-Whitney $U$ test $(P<0.05)$ were applied. The most observed species were found in sample FAF1 from Xingyi (1,022 species), whereas the lowest number was found in sample FBF1 (252 species). Sample FAF1 also contained a disproportionally high abundance of an OTU assigned to the genus Bolbitius (89.5\%). This is likely owing to a high occurrence of mycelium that was present in this sample; thus, the removal of this sample from the fungal library is recommended prior to deepening analyses. The fungal community structure was assessed at genus level owing to the lower diversity (Supplementary Figure S4). Among the other samples, Fusarium (12.3\% average abundance) was the predominant genus, followed by Mortierella (11.8\%), Gibberella (2.6\%), Clonostachys (2.3\%), and Ustilaginoidea (1.6\%).

qPCR-based detection of $\boldsymbol{P}$. nicotianae. A total of 11 soil samples contained detectable amounts of $P$. nicotianae DNA, and seven samples were below the detection level (Supplementary Table S1). The determined efficiency of the qPCR was $90.6 \%$ under the described 
conditions. There was no evident correlation between black shank disease occurrence in the tobacco fields and presence of pathogen DNA in the samples. Five of the disease-free field samples contained detectable amounts of $P$. nicotianae DNA. In contrast, three samples from fields with disease incidence were under the detection limit for the pathogen.

Physicochemical field soil properties. The field soils from the three sampling sites in Guizhou province are typical clay loam. They had a similar $\mathrm{pH}$ (6.41 to 6.93), cation exchange capacity (26.0 to $33.0 \mathrm{cmol} / \mathrm{kg}$ ), and water-soluble salts ( 1.80 to $2.10 \mathrm{~g} / \mathrm{kg})$; meanwhile, the total nitrogen (1.24 to $1.89 \%$ ), organic matter (2.74 to $4.68 \%$ ), and total potassium (315.86 to $593.92 \mathrm{mg} / \mathrm{kg}$ ) showed higher variations between the sites; a substantially higher total phosphate concentration was observed for site $A\left(1.07 \times 10^{3} \mathrm{mg} / \mathrm{kg}\right)$ compared with the other two sites (612.00 to $875.00 \mathrm{mg} / \mathrm{kg}$ ) (Supplementary Table S2).

\section{Future Directions}

The datasets that were made available in this resource announcement will be complemented with additional datasets obtained from $N$. tabacum fields affected by different plant pathogens (e.g., Ralstonia solanacearum and Meloidogyne incognita) in order to provide sufficient amplicon library sizes that will facilitate statistically verifiable correlation analyses. As parts of a multidisease correlation study, the datasets will be used to identify diseasespecific biomarkers in the bacteriome and mycobiome of soil samples. These biomarkers could provide a basis for early-stage disease detection and improved disease management strategies. Furthermore, we want to test if disease-specific biomarkers can also be implemented for the same purposes in other plants from the Solanaceae family.

\section{Availability of Data and Materials}

Both NGS libraries were publicly deposited at the Sequence Read Archive of the National Center for Biotechnology Information. The 16S rRNA gene fragment library (18 samples) and the fungal ITS library (18 samples) are both accessible with the required metadata under the study accession PRJNA528359. Additional metadata that are useful for further processing of the datasets are provided in Supplementary Table S1.

\section{Acknowledgments}

We thank Shuran Yu from Novogene (Beijing, China) for her support during the NGS analyses and data processing.

\section{Author Contributions}

$\mathrm{XC}, \mathrm{HY}$, and TC conceived the study. $\mathrm{XC}$ and $\mathrm{BH}$ obtained the samples from the tobacco cultivation fields in Guizhou province. BH conducted the DNA extractions, efficiency controls, and amplifications under guidance of TC. XC and TC conducted assessments of the bioinformatic data and wrote the manuscript with specific inputs from HY. All authors read and approved the final version of the manuscript.

\section{Literature Cited}

Bokulich, N. A., Subramanian, S., Faith, J. J., Gevers, D., Gordon, J. I., Knight, R., Mills, D. A., and Caporaso, J. G. 2013. Quality-filtering vastly improves diversity estimates from Illumina amplicon sequencing. Nat. Methods 10:57-59.

Edgar, R. C. 2013. UPARSE: Highly accurate OTU sequences from microbial amplicon reads. Nat. Methods 10:996-998.

Edgar, R. C., Haas, B. J., Clemente, J. C., Quince, C., and Knight, R. 2011. UCHIME improves sensitivity and speed of chimera detection. Bioinformatics 27:2194-2200.

Erwin, D. C., and Ribeiro, O. K. 1996. Phytophthora Diseases Worldwide. APS Press, St. Paul, MN.

Food and Agriculture Organization of the United Nations (FAO). 2019. FAOSTAT Statistics Database. Retrieved 21 March 2019 from http://www.fao.org/faostat/ en/\#data/QC/visualize.

Ginnan, N. A., Dang, T., Bodaghi, S., Ruegger, P. M., Peacock, B. B., McCollum, G., England, G., Vidalakis, G., Roper, C., Rolshausen, P., and Borneman, J. 2018.
Bacterial and fungal next generation sequencing datasets and metadata from citrus infected with 'Candidatus Liberibacter asiaticus'. Phytobiomes J. 2:64-70.

Groark, K. P. 2010. The angel in the gourd: Ritual, therapeutic, and protective uses of tobacco (Nicotiana tabacum) among the Tzeltal and Tzotzil Maya of Chiapas, Mexico. J. Ethnobiol. 30:5-30.

Haas, B. J., Gevers, D., Earl, A. M., Feldgarden, M., Ward, D. V., Giannoukos, G. Ciulla, D., Tabbaa, D., Highlander, S. K., Sodergren, E., Methe, B., DeSantis, T. Z., Petrosino, J. F., Knight, R., and Birren, B. W. 2011. Chimeric 16S rRNA sequence formation and detection in Sanger and 454-pyrosequenced PCR amplicons. Genome Res. 21:494-504.

Huang, J., Wu, J., Li, C., Xiao, C., and Wang, G. 2010. Detection of Phytophthora nicotianae in soil with real-time quantitative PCR. J. Phytopathol. 158:15-21.

Jiang, G., Wei, Z., Xu, J., Chen, H., Zhang, Y., She, X., Macho, A. P., Ding, W., and Liao, B. 2017. Bacterial wilt in China: History, current status, and future perspectives. Front. Plant Sci. 8:1549. 
Liu, X., Zhang, S., Jiang, Q., Bai, Y., Shen, G., Li, S., and Ding, W. 2016. Using community analysis to explore bacterial indicators for disease suppression of tobacco bacterial wilt. Sci. Rep. 6:36773.

Ma, L., Zhang, H. Y., Zhou, X. K., Yang, C. G., Zheng, S. C., Duo, J. L., and Mo, M. H. 2018. Biological control tobacco bacterial wilt and black shank and root colonization by bio-organic fertilizer containing bacterium Pseudomonas aeruginosa NXHG29. Appl. Soil Ecol. 129:136-144.

Magoč, T., and Salzberg, S. L. 2011. FLASH: Fast length adjustment of short reads to improve genome assemblies. Bioinformatics 27:2957-2963.

Panabieres, F., Ali, G. S., Allagui, M. B., Dalio, R. J. D, Gudmestad, N. C., Kuhn, M., Guha-Roy, S., Schena, L., and Zampounis, A. 2016. Phytophthora nicotianae diseases worldwide: New knowledge of a long-recognised pathogen. Phytopathol. Mediterr. 55:20-40.

Shen, G., Zhang, S., Liu, X., Jiang, Q., and Ding, W. 2018. Soil acidification amendments change the rhizosphere bacterial community of tobacco in a bacterial wilt affected field. Appl. Micobiol. Biot. 102:9781-9791.
Shew, H. D., and Lucas, G. B. 1991. Compendium of Tobacco Diseases. APS Press, St. Paul, MN.

Wang, H., Yang, S., Wang, M., Xia, H., Li, W., Zhang, H., Cao, Y., Lu, N., Shang, S., and Shi, J. 2013. Sensitivity of Phytophthora parasitica to mandipropamid: In vitro determination of baseline sensitivity and in vivo fungitoxicity. Crop Prot. 43:251-255

Wang, Q., Garrity, G. M., Tiedje, J. M., and Cole, J. R. 2007. Naive Bayesian classifier for rapid assignment of rRNA sequences into the new bacterial taxonomy. Appl. Environ. Microbiol. 73:5261-5267.

Wu, K., Yuan, S., Wang, L., Shi, J., Zhao, J., Shen, B., and Shen, Q. 2014. Effects of bio-organic fertilizer plus soil amendment on the control of tobacco bacterial wilt and composition of soil bacterial communities. Biol. Fertil. Soils 50: 961-971.

Yang, H., Li, J., Xiao, Y., Gu, Y., Liu, H., Liang, Y., Liu, X., Hu, J., Meng, D., and Yin, H. 2017. An integrated insight into the relationship between soil microbial community and tobacco bacterial wilt disease. Front. Microbiol. 8:2179. 